\title{
Association schemes on the Schubert cells of a Grassmannian
}

\author{
Yuta Watanabe
}

October 8, 2018

\begin{abstract}
Let $\mathbb{F}$ be any field. The Grassmannian $\operatorname{Gr}(m, n)$ is the set of $m$-dimensional subspaces in $\mathbb{F}^{n}$, and the general linear group $\mathrm{GL}_{n}(\mathbb{F})$ acts transitively on it. The Schubert cells of $\operatorname{Gr}(m, n)$ are the orbits of the Borel subgroup $\mathcal{B} \subset \mathrm{GL}_{n}(\mathbb{F})$ on $\operatorname{Gr}(m, n)$. We consider the association scheme on each Schubert cell defined by the $\mathcal{B}$-action and show it is symmetric and it is the generalized wreath product of one-class association schemes, which was introduced by R. A. Bailey [European Journal of Combinatorics 27 (2006) $428-435]$.
\end{abstract}

2010 Mathematics Subject Classification: 51E20; 14M15

Keywords: subspace lattice; Schubert cell; Borel subgroup; association scheme; generalized wreath product

\section{Introduction}

Let $n$ be a positive integer, and let $\mathbb{F}$ be any field. The subspace lattice $\mathcal{P}_{n}(\mathbb{F})$ (also known as the finite projective space) is the poset of linear subspaces of $\mathbb{F}^{n}$. The general linear group $\mathrm{GL}_{n}(\mathbb{F})$ acts on $\mathcal{P}_{n}(\mathbb{F})$. The natural grading structure of $\mathcal{P}_{n}(\mathbb{F})$ is given by $\mathrm{GL}_{n}(\mathbb{F})$-action, and each fiber (i.e., orbit) is called a Grassmannian. In other words, the $\operatorname{Grassmannian} \operatorname{Gr}(m, n)$ is the set of $m$-dimensional subspaces in $\mathbb{F}^{n}$, where $0 \leq m \leq n$. Let $\mathcal{B}$ denote the Borel subgroup of $\mathrm{GL}_{n}(\mathbb{F})$. Then, the $\mathcal{B}$-action defines a finer "hyper-cubic" grading structure of $\mathcal{P}_{n}(\mathbb{F})$, and each fiber contained in $\operatorname{Gr}(m, n)$ is called a Schubert cell of $\operatorname{Gr}(m, n)$. See [8] for details. The author showed in [15] that the algebra defined from the "hyper-cubic" grading structure of $\mathcal{P}_{n}(\mathbb{F})$ together with its incidence structure has a close relation to the quantum affine algebra $U_{q}\left(\widehat{\mathfrak{s l}}_{2}\right)$, if $\mathbb{F}$ is a finite field of $q^{2}$ elements. In this paper, we study the Schubert cells of a Grassmannian from the combinatorial point of view of association schemes. More precisely, we show that the association scheme defined by the $\mathcal{B}$-action on each Schubert cell is a generalized wreath product of one-class association schemes with the base set $\mathbb{F}$. The concept of a generalized wreath product of association schemes was introduced by R. A. Bailey [1] in 2006. The (usual) wreath product of association schemes has been actively studied (see 
e.g., [3 [5, 7, 10, 12, 13, 16]), and we may view the result of this paper as demonstrating the fundamental importance of Bailey's generalization as well.

Before the main discussion, we briefly recall the notion of the generalized wreath product of association schemes. For the definition of association schemes, see [2, 9, 17], and for the theory of posets, see [11]. Let $(X, \leq)$ be a nonempty finite poset. A subset $Y$ in $X$ is called an anti-chain if any two elements in $Y$ is incomparable. For an anti-chain $Y$ in $X$, define the down-set (also known as the order ideal) by

$$
\operatorname{Down}(Y)=\{x \in X \mid x<y \text { for some } y \in Y\}
$$

Note that this definition follows [1] and it is different from [11], where $\operatorname{Down}(Y) \cup Y$ is called the down-set of $Y$. For each $x \in X$, let $\mathcal{Q}_{x}$ denote an $r_{x}$-class association scheme on a set $\Omega_{x}$. We do not assume either $\mathcal{Q}_{x}$ is symmetric or $\Omega_{x}$ is finite. Let $R_{x, i}$ denote the $i$-th associate class for $i \in\left\{0,1, \ldots, r_{x}\right\}$. By convention, we choose the index so that $R_{x, 0}=\left\{(\omega, \omega) \mid \omega \in \Omega_{x}\right\}$. We set $\Omega=\prod_{x \in X} \Omega_{x}$. For each anti-chain $Y$ in $X$ and for each $\left(i_{x}\right)_{x \in Y} \in \prod_{x \in Y}\left\{1,2, \ldots, r_{x}\right\}$, let $R\left(Y,\left(i_{x}\right)_{x \in Y}\right)$ denote the set of $\left(\left(\alpha_{x}\right)_{x \in X},\left(\beta_{x}\right)_{x \in X}\right) \in \Omega \times \Omega$ satisfying (i) $\alpha_{x}=\beta_{x}$ if $x \in X \backslash(Y \cup \operatorname{Down}(Y))$, and (ii) $\left(\alpha_{x}, \beta_{x}\right) \in R_{x, i_{x}}$ if $x \in Y$. Let $\mathcal{R}$ denote the set of $R\left(Y,\left(i_{x}\right)_{x \in Y}\right)$ for all anti-chains $Y$ in $X$ and $\left(i_{x}\right)_{x \in Y} \in \prod_{x \in Y}\left\{1,2, \ldots, r_{x}\right\}$.

Theorem 1.1 (cf. [1, Theorem 3]). The pair $(\Omega, \mathcal{R})$ is an association scheme.

The association scheme $(\Omega, \mathcal{R})$ in Theorem 1.1 is called the generalized wreath product of $\mathcal{Q}_{x}$ over the poset $X$. We remark that Bailey [1, Theorem 3] assumes that each base set $\Omega_{x}$ is finite and each association scheme $\mathcal{Q}_{x}$ is symmetric. However, the theorem is still true if we drop both of these assumptions.

\section{Subspace lattices}

Throughout this paper, we fix a positive integer $n$ and a field $\mathbb{F}$. Let $\mathbb{F}^{n}$ denote the $n$ dimensional column vector space over $\mathbb{F}$. By the subspace lattice, denoted by $\mathcal{P}_{n}(\mathbb{F})$, we mean the poset consisting of all subspaces in $\mathbb{F}^{n}$ with partial order given by inclusion. We fix the sequence $\left\{V_{i}\right\}_{i=0}^{n}$ in $\mathcal{P}_{n}(\mathbb{F})$ such that each $V_{i}$ consists of vectors whose bottom $n-i$ entries are zero. We remark that $\left\{V_{i}\right\}_{i=0}^{n}$ is a (complete) flag (i.e., a maximal chain) in $\mathcal{P}_{n}(\mathbb{F})$.

Let $\operatorname{Mat}_{n}(\mathbb{F})$ denote the set of $n \times n$ matrices with entries in $\mathbb{F}$. Let $\mathrm{GL}_{n}(\mathbb{F})$ denote the set of invertible matrices in $\operatorname{Mat}_{n}(\mathbb{F})$. Observe that $\mathrm{GL}_{n}(\mathbb{F})$ acts on $\mathbb{F}^{n}$ by left multiplication, and hence it acts on $\mathcal{P}_{n}(\mathbb{F})$. Let $\mathcal{B}$ denote the (Borel) subgroup in $\mathrm{GL}_{n}(\mathbb{F})$ stabilizing $\left\{V_{i}\right\}_{i=0}^{n}$. In other words, $\mathcal{B}$ consists of all upper triangular invertible matrices in $\operatorname{Mat}_{n}(\mathbb{F})$. In this paper, we consider the $\mathcal{B}$-action on $\mathcal{P}_{n}(\mathbb{F})$.

A matrix in $\operatorname{Mat}_{n}(\mathbb{F})$ is said to be in reverse column echelon form if the following two conditions are met:

(CE1) Any zero columns are right of all nonzero columns.

(CE2) The last nonzero entry of a nonzero column is always strictly below of the last nonzero entry of its right column. 
A matrix in $\operatorname{Mat}_{n}(\mathbb{F})$ is said to be in reduced reverse column echelon form if it is in reverse column echelon form and the following third condition is also met:

(CE3) Every last nonzero entry of a nonzero column is 1 and is the only nonzero entry in its row.

By elementary linear algebra, we have the following.

Proposition 2.1. There exists a bijection between the following two sets:

(i) the set of all matrices in $\operatorname{Mat}_{n}(\mathbb{F})$ in reduced reverse column echelon form,

(ii) the set $\mathcal{P}_{n}(\mathbb{F})$ of all subspaces in $\mathbb{F}^{n}$,

that sends a matrix in $\operatorname{Mat}_{n}(\mathbb{F})$ to its column space.

Proof. See for instance [6].

Example 2.2 $(n=7)$. Let $e_{1}, e_{2}, \ldots, e_{7}$ denote the standard basis for $\mathbb{F}^{7}$. Suppose $U$ is the 4-dimensional subspace in $\mathbb{F}^{7}$ given by $U=\operatorname{Span}\left\{8 e_{1}+6 e_{3}+4 e_{6}+2 e_{7}, 8 e_{1}+9 e_{3}+e_{4}+e_{5}, 4 e_{1}+\right.$ $\left.e_{2}+5 e_{3}+e_{4}, 3 e_{1}+e_{2}\right\}$. Then the following is the matrix corresponding to $U$ by the bijection in Proposition 2.1:

$$
M=\left(\begin{array}{lllllll}
4 & 7 & 1 & 3 & 0 & 0 & 0 \\
0 & 0 & 0 & 1 & 0 & 0 & 0 \\
3 & 4 & 5 & 0 & 0 & 0 & 0 \\
0 & 0 & 1 & 0 & 0 & 0 & 0 \\
0 & 1 & 0 & 0 & 0 & 0 & 0 \\
2 & 0 & 0 & 0 & 0 & 0 & 0 \\
1 & 0 & 0 & 0 & 0 & 0 & 0
\end{array}\right)
$$

Observe that for $M, N \in \operatorname{Mat}_{n}(\mathbb{F})$ in reduced reverse column echelon form and for $G \in \mathcal{B}$, the column space of $N$ moves to that of $M$ by the $G$-action if and only if $M$ and $G N$ are column equivalent. Since $G N$ is in reverse column echelon form (but not necessarily reduced), these conditions are equivalent to $M=G N H^{T}$ for some $H \in \mathcal{B}$. For notational convenience, we write $M \sim N$ if there exist $G, H \in \mathcal{B}$ such that $M=G N H^{T}$. Observe that $\sim$ is an equivalence relation on $\operatorname{Mat}_{n}(\mathbb{F})$.

For the rest of this paper, we will identify $\mathcal{P}_{n}(\mathbb{F})$ with the set of all matrices in $\operatorname{Mat}_{n}(\mathbb{F})$ in reduced reverse column echelon form by the bijection in Proposition 2.1.

\section{The $\mathcal{B}$-action on $\mathcal{P}_{n}(\mathbb{F})$}

For a positive integer $m$, we write $[m]=\{1,2, \ldots, m\}$. We define a partial order in the index set $[n] \times[n]$ of matrices in $\operatorname{Mat}_{n}(\mathbb{F})$ by $(i, j) \leq(k, l)$ if $i \leq k$ and $j \leq l$. This is known as the direct product order in [11, Section 3.2]. For $M \in \operatorname{Mat}_{n}(\mathbb{F})$, by the support of $M$, denoted by $\operatorname{Supp}(M)$, we mean the subposet of $[n] \times[n]$ consisting of all indices $(i, j) \in[n] \times[n]$ with $M_{i, j} \neq 0$. The pivot-set of $M$, denoted by $\operatorname{Piv}(M)$, is the set of all maximal elements in 
$\operatorname{Supp}(M)$. Each element in the pivot-set is called a pivot. Observe that $(i, j) \in \operatorname{Piv}(M)$ if and only if $M_{i, j} \neq 0$ and $M_{k, l}=0$ if $(k, l)>(i, j)$. We remark that every entry indexed by a pivot of a matrix in $\mathcal{P}_{n}(\mathbb{F})$ must be 1 by the condition (CE3).

Lemma 3.1. For $M, N \in \mathcal{P}_{n}(\mathbb{F})$, the following are equivalent:

(i) $M \sim N$

(ii) $\operatorname{Piv}(M)=\operatorname{Piv}(N)$.

Proof. (i) $\Rightarrow$ (ii) Suppose $M \sim N$. There exist $G, H \in \mathcal{B}$ such that $M=G N H^{T}$. It suffices to show $\operatorname{Piv}(N) \subseteq \operatorname{Piv}(M)$. For $(i, j) \in \operatorname{Piv}(N)$, we have $N_{i, j}=1$ and $N_{k, l}=0$ if $(k, l)>(i, j)$. Since $G, H$ are upper triangular, we have

$$
M_{k, l}=\sum_{s=k}^{n} \sum_{t=l}^{n} G_{k, s} N_{s, t} H_{l, t}= \begin{cases}G_{i, i} H_{j, j} & \text { if }(k, l)=(i, j), \\ 0 & \text { if }(k, l)>(i, j) .\end{cases}
$$

Since $G, H$ are invertible, $G_{i, i} H_{j, j} \neq 0$. These imply $(i, j) \in \operatorname{Piv}(M)$ and hence $\operatorname{Piv}(N) \subseteq$ $\operatorname{Piv}(M)$.

(ii) $\Rightarrow$ (i) Suppose $\operatorname{Piv}(M)=\operatorname{Piv}(N)$. Take $X \in \mathcal{P}_{n}(\mathbb{F})$ with $X_{i, j}=1$ if $(i, j) \in \operatorname{Piv}(M)$ and $X_{i, j}=0$ otherwise. Observe that for each $j \in[n]$, there exists at most one $k$ such that $(j, k) \in \operatorname{Piv}(M)$ and then we $\operatorname{define} G \in \operatorname{Mat}_{n}(\mathbb{F})$ by

$$
G_{i, j}= \begin{cases}M_{i, k} & \text { if }(j, k) \in \operatorname{Piv}(M) \text { for some } k, \\ \delta_{i, j} & \text { if there is no } k \text { such that }(j, k) \in \operatorname{Piv}(M)\end{cases}
$$

for $i, j \in[n]$. Then we have $G_{i, i}=1$ for $i \in[n]$ and $G_{i, j}=0$ if $j<i$ for $i, j \in[n]$. Thus $G \in \mathcal{B}$. By the direct calculation, we have $M=G X$ and hence, $M \sim X$. Similarly we have $N \sim X$ and so $M \sim N$.

Let $1 \leq m \leq n-1$ and $M \in \mathcal{P}_{n}(\mathbb{F})$ with $\operatorname{rank} M=m$. Note that we avoid the trivial cases $m=0$ and $m=n$. Since the pivots of $M$ lie in the first $m$ columns, $\operatorname{Piv}(M)$ is an anti-chain in $[n] \times[m]$ of size $m$. For $1 \leq m \leq n-1$ and for an anti-chain $\alpha$ in $[n] \times[m]$ of size $m$, we set

$$
\mathcal{O}_{\alpha}=\left\{M \in \mathcal{P}_{n}(\mathbb{F}) \mid \operatorname{Piv}(M)=\alpha\right\} .
$$

For each $1 \leq m \leq n-1$ and each anti-chain $\alpha$ in $[n] \times[m]$ of size $m$, consider $M \in \mathcal{P}_{n}(\mathbb{F})$ with $M_{i, j}=1$ if $(i, j) \in \alpha$ and $M_{i, j}=0$ otherwise. Then we have $M \in \mathcal{O}_{\alpha}$ and in particular $\mathcal{O}_{\alpha} \neq \emptyset$.

Proposition 3.2. The rank of any matrix in (1) is $m=|\alpha|$. Moreover, each subset (11) is an orbit of the $\mathcal{B}$-action on $\mathcal{P}_{n}(\mathbb{F})$.

Proof. Immediate from the construction and Lemma 3.1. 
Recall the Grassmannian $\operatorname{Gr}(m, n)$ and we identify $\operatorname{Gr}(m, n)$ with a set of matrices by the bijection in Proposition 2.1. In other words,

$$
\operatorname{Gr}(m, n)=\left\{M \in \mathcal{P}_{n}(\mathbb{F}) \mid \operatorname{rank} M=m\right\} .
$$

By Proposition 3.2, each $\mathcal{O}_{\alpha}$ in (11) is a $\mathcal{B}$-orbit in $\operatorname{Gr}(m, n)$, where $m=|\alpha|$. Thus, it is called a Schubert cell of a Grassmannian [8].

Example $3.3(n=7, m=4)$. Take $M \in \mathcal{P}_{7}(\mathbb{F})$ as in Example 2.2. Then we have $\operatorname{Piv}(M)=$ $\{(2,4),(4,3),(5,2),(7,1)\}$. Moreover, $\mathcal{O}_{\operatorname{Piv}(M)}$ is the set of matrices of the form

$$
\left(\begin{array}{lllllll}
* & * & * & * & 0 & 0 & 0 \\
0 & 0 & 0 & 1 & 0 & 0 & 0 \\
* & * & * & 0 & 0 & 0 & 0 \\
0 & 0 & 1 & 0 & 0 & 0 & 0 \\
0 & 1 & 0 & 0 & 0 & 0 & 0 \\
* & 0 & 0 & 0 & 0 & 0 & 0 \\
1 & 0 & 0 & 0 & 0 & 0 & 0
\end{array}\right),
$$

where the symbol $*$ denotes an arbitrary element in $\mathbb{F}$.

Lemma 3.4. Let $1 \leq m \leq n-1$ and let $\alpha$ denote an anti-chain in $[n] \times[m]$ of size $m$. For $M, N, M^{\prime}, N^{\prime} \in \mathcal{O}_{\alpha}$, the following are equivalent:

(i) $(M, N)$ moves to $\left(M^{\prime}, N^{\prime}\right)$ by the diagonal $\mathcal{B}$-action,

(ii) $\operatorname{Piv}(M-N)=\operatorname{Piv}\left(M^{\prime}-N^{\prime}\right)$.

Proof. (i) $\Rightarrow$ (ii) Suppose there exist $G, H, K \in \mathcal{B}$ such that $M^{\prime}=G M H^{T}$ and $N^{\prime}=G N K^{T}$. Then we have

$$
\begin{gathered}
\operatorname{Piv}(G M)=\operatorname{Piv}(G N)=\alpha, \\
\operatorname{Piv}(G M-G N)=\operatorname{Piv}(M-N)
\end{gathered}
$$

since $G \in \mathcal{B}$ (cf. Lemma [3.1). We write $\alpha=\left\{\left(k_{r}, r\right) \mid r \in[m]\right\}$ and observe that $k_{1}>k_{2}>$ $\cdots>k_{m}$ and that $\operatorname{Piv}(M-N) \subseteq \operatorname{Down}(\alpha)$.

Take $(i, j) \in \operatorname{Piv}(M-N)$. Observe that $j \in[m]$ and $k_{j}>i$ and hence there exists $m^{\prime}=\max \left\{r \in[m] \mid k_{r}>i\right\}$. For $r, l \in[m]$ with $j \leq r \leq m^{\prime}$ and $j \leq l \leq m^{\prime}$, since $H, K$ are upper triangular, we have

$$
\begin{aligned}
M_{k_{r}, l}^{\prime} & =\sum_{t=l}^{n}(G M)_{k_{r}, t} H_{l, t}, \\
N_{k_{r}, l}^{\prime} & =\sum_{t=l}^{n}(G N)_{k_{r}, t} K_{l, t} .
\end{aligned}
$$

In the above equations, we have the following: For each $r, l$, we have $M_{k_{r}, l}^{\prime}=N_{k_{r}, l}^{\prime}=\delta_{r, l}$ by (CE3); For each $r, t$, we have $(G M)_{k_{r}, t}=(G N)_{k_{r}, t}$ since $\left(k_{r}, t\right)>(i, j)$ and by (4)); For each 
$r, t$ with $r<t$, we have $(G M)_{k_{r}, r}=(G N)_{k_{r}, r}=G_{k_{r}, k_{r}} \neq 0$ and $(G M)_{k_{r}, t}=(G N)_{k_{r}, t}=0$ since $\left(k_{r}, r\right) \in \alpha$ and by (3). By these comments, for each $l \in[m]$ with $j \leq l \leq m^{\prime}$, both $\left(H_{l, l}, H_{l, l+1}, \ldots, H_{l, m^{\prime}}\right)$ and $\left(K_{l, l}, K_{l, l+1}, \ldots, K_{l, m^{\prime}}\right)$ are solutions to the same system of $m^{\prime}-j+1$ independent linear equations. Hence, $H_{l, t}=K_{l, t}$ for $j, t \in[m]$ with $j \leq l \leq t \leq m^{\prime}$.

For $(k, l) \in[n] \times[n]$ with $(k, l)>(i, j)$, we have $(G M)_{k, t}=(G N)_{k, t}$ if $t \geq l$ since $(k, t)>(i, j)$ and by (4), and we also have $(G M)_{k, t}=(G N)_{k, t}=0$ if $t>m^{\prime}$ by the definition of $m^{\prime}$ and by (3). Recall that we have shown $H_{l, t}=K_{l, t}$ if $j \leq l \leq t \leq m^{\prime}$. By these comments, we have

$$
M_{k, l}^{\prime}=\sum_{t=l}^{n}(G M)_{k, t} H_{l, t}=\sum_{t=l}^{n}(G N)_{k, t} K_{l, t}=N_{k, l}^{\prime} .
$$

Similarly, we have

$$
M_{i, j}^{\prime}-N_{i, j}^{\prime}=\sum_{t=j}^{n}(G M)_{i, t} H_{j, t}-\sum_{t=j}^{n}(G N)_{i, t} K_{j, t}=\left((G M)_{i, j}-(G N)_{i, j}\right) H_{j, j} .
$$

In the above equations, we have $(G M)_{i, j} \neq(G N)_{i, j}$ by (44), and we also have $H_{j, j} \neq 0$ since $H \in \mathcal{B}$. Therefore we obtain $M_{i, j}^{\prime} \neq N_{i, j}^{\prime}$. These imply $(i, j) \in \operatorname{Piv}\left(M^{\prime}-N^{\prime}\right)$ and hence $\operatorname{Piv}(M-N) \subseteq \operatorname{Piv}\left(M^{\prime}-N^{\prime}\right)$. Since $G, H, K$ are invertible, we also have $\operatorname{Piv}\left(M^{\prime}-N^{\prime}\right) \subseteq$ $\operatorname{Piv}(M-N)$. Consequently, we have $\operatorname{Piv}(M-N)=\operatorname{Piv}\left(M^{\prime}-N^{\prime}\right)$.

(ii) $\Rightarrow$ (i) Let $M, N, M^{\prime}, N^{\prime} \in \mathcal{O}_{\alpha}$ with $\operatorname{Piv}(M-N)=\operatorname{Piv}\left(M^{\prime}-N^{\prime}\right)$. Take $X \in \mathcal{O}_{\alpha}$ with $X_{i, j}=1$ if $(i, j) \in \alpha \cup \operatorname{Piv}(M-N)$ and $X_{i, j}=0$ otherwise, and $Y \in \mathcal{O}_{\alpha}$ with $Y_{i, j}=1$ if $(i, j) \in \alpha$ and $Y_{i, j}=0$ otherwise. Define $G \in \operatorname{Mat}_{n}(\mathbb{F})$ by

$$
G_{i, j}= \begin{cases}N_{i, k} & \text { if }(j, k) \in \alpha \text { for some } k, \\ M_{i, k}-N_{i, k} & \text { if }(j, k) \in \operatorname{Piv}(M-N) \text { for some } k, \\ \delta_{i, j} & \text { if there is no } k \text { such that }(j, k) \in \alpha \cup \operatorname{Piv}(M-N)\end{cases}
$$

for $i, j \in[n]$. Then we have $G \in \mathcal{B}$ and $M=G X$ and $N=G Y$. Similarly there exists $G^{\prime} \in \mathcal{B}$ such that $M^{\prime}=G^{\prime} X$ and $N^{\prime}=G^{\prime} Y$. Therefore $(M, N)$ moves to $\left(M^{\prime}, N^{\prime}\right)$ by the diagonal action of $G^{\prime} G^{-1}$.

Let $1 \leq m \leq n-1$ and let $\alpha$ denote an anti-chain in $[n] \times[m]$ of size $m$. Let $M, N \in \mathcal{O}_{\alpha}$. Observe that $\operatorname{Piv}(M-N)$ is an anti-chain in

$$
\mathcal{D}(\alpha)=\{(i, j) \in \operatorname{Down}(\alpha) \mid \text { there is no } k \text { such that }(i, k) \in \alpha\} .
$$

For $1 \leq m \leq n-1$ and for an anti-chain $\alpha$ in $[n] \times[m]$ of size $m$ and for an anti-chain $\beta$ in $\mathcal{D}(\alpha)$, we set

$$
\mathcal{R}_{\alpha, \beta}=\left\{(M, N) \in \mathcal{O}_{\alpha} \times \mathcal{O}_{\alpha} \mid \operatorname{Piv}(M-N)=\beta\right\} .
$$

For each $1 \leq m \leq n-1$ and each anti-chain $\alpha$ in $[n] \times[m]$ of size $m$ and each anti-chain $\beta$ in $\mathcal{D}(\alpha)$, consider $M \in \mathcal{P}_{n}(\mathbb{F})$ with $M_{i, j}=1$ if $(i, j) \in \alpha \cup \beta$ and $M_{i, j}=0$ otherwise, and $N \in \mathcal{P}_{n}(\mathbb{F})$ with $N_{i, j}=1$ if $(i, j) \in \alpha$ and $N_{i, j}=0$ otherwise. Then we have $(M, N) \in \mathcal{R}_{\alpha, \beta}$ and in particular $\mathcal{R}_{\alpha, \beta} \neq \emptyset$. 
Proposition 3.5. Let $1 \leq m \leq n-1$ and let $\alpha$ denote an anti-chain in $[n] \times[m]$ of size $m$. Each subset (6) is an orbital of the $\mathcal{B}$-action on $\mathcal{O}_{\alpha}$.

Proof. Immediate from Lemma 3.4 .

Let $1 \leq m \leq n-1$. For an anti-chain $\alpha$ in $[n] \times[m]$ of size $m$, consider

$$
\mathcal{D}_{1}(\alpha)=\{i \mid \text { there is no } k \text { such that }(i, k) \in \alpha\} \text {. }
$$

Then we have $\left|\mathcal{D}_{1}(\alpha)\right|=n-m$ since $|\alpha|=m$. For $1 \leq i \leq n-m$, we define $\lambda_{i}=\mid\left\{j \mid\left(d_{i}, j\right) \in\right.$ $\mathcal{D}(\alpha)\} \mid$, where $d_{i}$ denotes the $i$-th smallest element in $\mathcal{D}_{1}(\alpha)$. Then $\lambda=\left(\lambda_{1}, \lambda_{2}, \ldots, \lambda_{n-m}\right) \in$ $\mathbb{N}^{n-m}$ is an integer partition (i.e., a non-increasing sequence) with largest part at most $m$, where

$$
\mathbb{N}=\{0,1, \ldots\} \text {. }
$$

Consider the map $\varphi_{m}$ which sends $\alpha$ to $\lambda$.

For an integer partition $\lambda=\left(\lambda_{1}, \lambda_{2}, \ldots, \lambda_{l}\right)$, the Ferrers board of shape $\lambda$ is defined by

$$
\left\{(i, j) \in \mathbb{N} \times \mathbb{N} \mid 1 \leq i \leq l, 1 \leq j \leq \lambda_{i}\right\}
$$

We endow the Ferrers board with direct product order in $\mathbb{N} \times \mathbb{N}$.

Lemma 3.6. For $1 \leq m \leq n-1$, the map $\varphi_{m}$ is a bijection between the following two sets:

(i) the anti-chains in $[n] \times[m]$ of size $m$;

(ii) the integer partitions in $\mathbb{N}^{n-m}$ with largest part at most $m$.

Proof. Let $1 \leq m \leq n-1$. It is clear that $\varphi_{m}$ is a map from (i) to (ii). We define the map $\varphi_{m}^{\prime}$ from (ii) to (i) as follows. For a given integer partition $\lambda$ in $\mathbb{N}^{n-m}$ with largest part at most $m$, we define $\alpha$ as the set of maximal elements in the Ferrers board of shape $\mu=\lambda \cup(m, m-1, \ldots, 1)$, which is the integer partition obtained by rearranging parts of both $\lambda$ and $(m, m-1, \ldots, 1)$ in non-increasing order. Since $\mu$ is in $\mathbb{N}^{n}$ and its largest part is $m, \alpha$ is an anti-chain in $[n] \times[m]$ of size $m$. The map $\varphi_{m}^{\prime}$ is defined to send $\lambda$ to $\alpha$. By construction, $\varphi_{m}$ and $\varphi_{m}^{\prime}$ are inverses and hence bijections.

Lemma 3.7. Let $1 \leq m \leq n-1$ and let $\alpha$ denote an anti-chain in $[n] \times[m]$ of size $m$. The poset $\mathcal{D}(\alpha)$ in (15) is isomorphic to the Ferrers board of shape $\varphi_{m}(\alpha)$. Moreover, there is a one-to-one correspondence between the anti-chains in $\mathcal{D}(\alpha)$ and the subpartitions of $\varphi_{m}(\alpha)$.

Proof. Recall the set $\mathcal{D}_{1}(\alpha)$ in (7). Then define the map $\psi$ from the Ferrers board of shape $\varphi_{m}(\alpha)$ to $\mathcal{D}(\alpha)$ by $\psi(i, j)=\left(d_{i}, j\right)$, where $d_{i}$ denotes the $i$-th smallest element in $\mathcal{D}_{1}(\alpha)$. It is obvious that $\psi$ is an order-preserving bijection. The first assertion follows. To show the second assertion, we define the map $\rho$ from the subpartitions of $\varphi_{m}(\alpha)$ to the anti-chains in $\mathcal{D}(\alpha)$ by $\rho(\mu)=\psi(\max (\mu))$, where $\max (\mu)$ is the set of all maximal elements in the Ferrers board of shape $\mu$. From the construction, the map $\rho$ is also a bijection. The second assertion follows.

Example $3.8(n=7, m=4)$. Take the anti-chain $\alpha=\{(2,4),(4,3),(5,2),(7,1)\}$ as in Example 3.3. Recall that $\mathcal{O}_{\alpha}$ is the set of matrices of the form (2). Then $\varphi_{4}(\alpha)=(4,3,1)$. We remark that each number in $(4,3,1)$ equals the number of $*$ 's in each row without a pivot. 


\section{The association scheme on each Schubert cell}

For $1 \leq m \leq n-1$ and for an anti-chain $\alpha$ in $[n] \times[m]$ of size $m$, by Propositions 3.2 and 3.5, the pair

$$
\mathfrak{X}_{\alpha}=\left(\mathcal{O}_{\alpha},\left\{\mathcal{R}_{\alpha, \beta}\right\}_{\beta}\right)
$$

becomes an association scheme, where $\beta$ runs over all anti-chains in $\mathcal{D}(\alpha)$ in (15). See [17, Preface]. We remark that by Lemma 3.6, the family of association schemes $\left\{\mathfrak{X}_{\alpha}\right\}_{\alpha}$ can be indexed by integer partitions $\lambda \in \mathbb{N}^{n-m}$ with largest part at most $m$. In this case, the associate classes of $\mathfrak{X}_{\alpha}=\mathfrak{X}_{\lambda}$ are indexed by the subpartitions of $\lambda$ by Lemma 3.7 .

Theorem 4.1. Let $1 \leq m \leq n-1$ and let $\alpha$ denote an anti-chain in $[n] \times[m]$ of size $m$. The association scheme $\mathfrak{X}_{\alpha}$ in (8) is symmetric.

Proof. Immediate from the definition of $\mathcal{R}_{\alpha, \beta}$.

Theorem 4.2. For $1 \leq m \leq n-1$ and for an anti-chain $\alpha$ in $[n] \times[m]$ of size $m$, the association scheme $\mathfrak{X}_{\alpha}$ in (8) is the generalized wreath product of the one-class association schemes with the base set $\mathbb{F}$ over the poset $\mathcal{D}(\alpha)$ in (5).

Proof. For $M, N \in \mathcal{O}_{\alpha}$ and for an anti-chain $\beta$ in $\mathcal{D}(\alpha)$, we have $(M, N) \in \mathcal{R}_{\alpha, \beta}$ if and only if $M_{i, j}=N_{i, j}$ if $(i, j) \notin \beta \cup \operatorname{Down}(\beta), M_{i, j} \neq N_{i, j}$ if $(i, j) \in \beta$. Therefore, this associate relation is the same as that of the generalized wreath product of the one-class association schemes with the base set $\mathbb{F}$ over the poset $\mathcal{D}(\alpha)$. So the result follows.

\section{Concluding remarks}

This paper focuses on the Schubert cells of a Grassmannian. It would be an interesting problem to find similar results on Schubert cells for other types of BN-pairs.

The Terwilliger algebra, introduced by P. Terwilliger [14], of the wreath product of oneclass association schemes is discussed in several papers [4, 12, 16]. We will consider the

Terwilliger algebra of the generalized wreath product of one-class association schemes in a future paper.

\section{Acknowledgments}

The author gratefully acknowledges the many helpful suggestions of his advisor, Hajime Tanaka during the preparation of the paper. The author thanks Paul Terwilliger for giving valuable comments and Motohiro Ishii for drawing the author's attention to the theory of Schubert cells. 


\section{References}

[1] R. A. Bailey, Generalized wreath products of association schemes, European J. Combin. 27 (2006), no. 3, 428-435.

[2] Eiichi Bannai and Tatsuro Ito, Algebraic combinatorics. I: Association schemes, The Benjamin/Cummings Publishing Co., Inc., Menlo Park, CA, 1984.

[3] Gargi Bhattacharyya, Terwilliger algebras of wreath products of association schemes, Thesis (Ph.D.)-Iowa State University, 2008.

[4] Gargi Bhattacharyya, Sung Y. Song, and Rie Tanaka, Terwilliger algebras of wreath products of one-class association schemes, J. Algebraic Combin. 31 (2010), no. 3, 455466.

[5] Akihide Hanaki and Kaoru Hirotsuka, Irreducible representations of wreath products of association schemes, J. Algebraic Combin. 18 (2003), no. 1, 47-52.

[6] Leslie Hogben (ed.), Handbook of linear algebra, 2nd ed., Discrete Mathematics and its Applications (Boca Raton), CRC Press, Boca Raton, FL, 2014.

[7] Kijung Kim, Terwilliger algebras of wreath products by quasi-thin schemes, Linear Algebra Appl. 437 (2012), no. 11, 2773-2780.

[8] V. Lakshmibai and Justin Brown, Flag varieties: An interplay of geometry, combinatorics, and representation theory, Texts and Readings in Mathematics, vol. 53, Hindustan Book Agency, New Delhi, 2009.

[9] William J. Martin and Hajime Tanaka, Commutative association schemes, European J. Combin. 30 (2009), no. 6, 1497-1525.

[10] Sung Y. Song, Bangteng Xu, and Shenglin Zhou, Combinatorial extensions of Terwilliger algebras and wreath products of association schemes, Discrete Math. 340 (2017), no. 5, 892-905.

[11] Richard P. Stanley, Enumerative combinatorics. Volume 1, 2nd ed., Cambridge Studies in Advanced Mathematics, vol. 49, Cambridge University Press, Cambridge, 2012.

[12] Rie Tanaka, Classification of commutative association schemes with almost commutative Terwilliger algebras, J. Algebraic Combin. 33 (2011), no. 1, 1-10.

[13] Rie Tanaka and Paul-Hermann Zieschang, On a class of wreath products of hypergroups and association schemes, J. Algebraic Combin. 37 (2013), no. 4, 601-619.

[14] Paul Terwilliger, The subconstituent algebra of an association scheme. I, J. Algebraic Combin. 1 (1992), no. 4, 363-388. 
[15] Yuta Watanabe, An algebra associated with a flag in a subspace lattice over a finite field and the quantum affine algebra $U_{q}\left(\widehat{\mathfrak{s l}}_{2}\right)$ (2017), available at arXiv:1709.06329[math.CO].

[16] Bangteng Xu, Characterizations of wreath products of one-class association schemes, J. Combin. Theory Ser. A 118 (2011), no. 7, 1907-1914.

[17] Paul-Hermann Zieschang, Theory of association schemes, Springer Monographs in Mathematics, Springer-Verlag, Berlin, 2005.

Yuta Watanabe

Graduate School of Information Sciences

Tohoku University

Sendai, 980-8579 Japan

email: watanabe@ims.is.tohoku.ac.jp 